\title{
Investigation of the drug distribution and release characteristics from particulate membranes
}

\author{
Tai-Horng Young ${ }^{\mathrm{a}, *}$, Wen-Yuan Chuang ${ }^{\mathrm{a}}$, Cheng-Wey Wei ${ }^{\mathrm{a}}$, Chih-Yuan Tang ${ }^{\mathrm{b}}$ \\ a Institute of Biomedical Engineering, College of Medicine and College of Engineering, \\ National Taiwan University, Taipei, Taiwan, ROC \\ ${ }^{\mathrm{b}}$ Advanced Instrumentation Center, National Taiwan University, Taipei, Taiwan, ROC
}

Received 29 October 1999; accepted 24 April 2001

\begin{abstract}
A new type of membrane structure has been developed for drug delivery that offers a biphasic release behavior. A mixture of DMSO, poly(ethylene-vinyl alcohol) (EVAL) and drug was precipitated from 1-octanol to form membranes by constituent particles bonded to each other. The drug can be distributed between particles or trapped in the particles during membrane formation, thereby allowing control of release kinetics. The drug release profile from the EVAL membrane was investigated using water as a release medium. Doxorubicin was used as a model drug because a hydrophilic drug can release in water but is only slightly dissolved in 1-octanol during membrane formation. It was found doxorubicin from the EVAL membrane showed a two-step release behavior. The drug release in the first step was rapid. This is probably due to the membrane is macroscopically porous that drug distributing outside the particles diffuses out rapidly. On the contrary, the drug release in the second step was slow and prolonged. This is probably due to drug entrapped in the particle during membrane formation. However, the particle structure is microscopically dense enough to increase the diffusion resistance, thus delaying and lowering the drug release rate. Therefore, such a membrane could be useful for sustained release of hydrophilic drugs. In addition, a mathematical mass transfer model for a two-step release of doxorubicin from the particulate membrane was developed and applied to the analysis of the drug release data. It can be found that the spherical particles encapsulated more than 55\% of the doxorubicin and had a very small mass transfer coefficient. These findings suggest that a drug delivery system using a particulate membrane is a promising method but the technique for loading drug in the particle requires further modification. (c) 2001 Elsevier Science B.V. All rights reserved.
\end{abstract}

Keywords: Particulate membranes; Doxorubicin; Drug delivery; Mass transfer model

\section{Introduction}

Since the development of asymmetric type membrane by Loeb and Sourirajan [1], the field of membrane science has experienced a notable growth. Various novel membranes have been tailored to fulfill

\footnotetext{
* Corresponding author. Tel.: +886-2-23970800/ext. 1455; fax: +886-2-23940049.

E-mail address: thyoung@ha.mc.ntu.edu.tw (T.-H. Young).
}

specific purposes and many of them have been commercialized in different phases of biomedical industry, e.g. controlled release [2] and hemodialysis [3]. In our laboratory, we have prepared poly(ethylene-vinyl alcohol) (EVAL) membranes characterized by a packed bed of particles [4-6]. Unlike the traditional asymmetric membrane, this membrane is virtually skinless. It is composed of nearly equal-sized spherical particles that interconnect to form a uniform structure. Such particulate 
membranes have attracted special attention among our research team and proved useful in microfiltration [4], plasma protein separation [5], and cell culture [6]. In previous studies, it was proposed that crystallization of polymer is responsible for the formation of particulate morphology [7-13].

The goal of the present study is to prepare particulate membranes as drug carrier for controlled drug release. The model drug used for the release experiments was doxorubucin. Doxorubicin was selected since it is a potent antineoplastic agent, used either as the sole agent or in combination chemotherapy against solid tumors. In this type of drug delivery system formulated as a particulate membrane, the drug reservoir is prepared by homogeneously dispersing drug and polymer in a common solvent [14], followed by precipitating this mixture in 1-octanol to form membranes by constituent particles bonded to each other. Drug may distribute between particles or within the particles during membrane formation, thereby allowing modification of drug release kinetics. It is well known that the success of the treatment of neoplastic diseases depends not only on the antineoplastic drug used but also on the method of administration. Therefore, if drug delivery from particulate membranes can change pharmacokinetics of the agents used, then it may reduce toxic side effects and increase efficacy of the therapy [15]. Furthermore, we took a mass transfer model to evaluate the drug release data. It was found that the spherical particles encapsulated more than $55 \%$ of the doxorubicin and had a sustained drug release for extended periods. Hence, this study developed an easy method for achieving a predictable and more accurate control of drug release kinetics.

\section{Materials and methods}

\subsection{Materials}

Membranes were prepared by using EVAL polymer (105A, Kuraray Co. Ltd., Japan) having an average ethylene content of $44 \mathrm{~mol} \%$. DMSO and 1-octanol of extra pure reagent grade (Nacalai Tesque, Kyoto, Japan) were used as received. Doxorubicin was purchased from Farmitalia Corporation (Italy) and used as received. Water was double distilled and de-ionized before used.

\subsection{Membrane preparation}

Particulate membranes were fabricated from the EVAL polymer by using the phase inversion method [16]. Thus, $2.5 \mathrm{~g}$ of EVAL was dissolved in $7.5 \mathrm{ml}$ of DMSO. To this solution $1 \mathrm{mg}$ of doxorubicin, predissolved in $0.5 \mathrm{ml}$ of DMSO, was added and mixed well. The resulting solution was dispersed uniformly on a glass plate (ca. $100 \mu \mathrm{m})$ using an autocoater (KCC303, RK Print-Coat Instruments, UK). Subsequently, the casting solution was quickly immersed into a 1-octanol bath for $1 \mathrm{~h}$ at room temperature, yielding a particulate membrane. For comparison of drug release behavior, a dense membrane blended with doxorubicin was prepared using solvent evaporation in a vacuum oven at $80^{\circ} \mathrm{C}$ for 2 days. The morphologies of the membranes were examined using a scanning electron microscope (SEM). The freeze-dried samples were gold coated and viewed by SEM (S-800, Hitachi, Japan) at $20 \mathrm{kV}$.

\subsection{Drug release study}

The release of doxorubicin from membranes into the medium (water) was carried out in 24-welled tissue culture polystyrene plates (Costar, USA) shaken by a rotator (DSR 2800A, Digisystem Laboratory Instruments, Taiwan) at a shaking speed of $100 \mathrm{rpm}$ at $37^{\circ} \mathrm{C}$. At first, 1-octanol was removed from the membrane under a vacuum condition of $24 \mathrm{~h}$ to prevent doxorubicin loss. Then the evaporated membranes (diameter $1.5 \mathrm{~cm}$ ) were placed in the bottom of wells of 24-well tissue culture plates by placing a sterilized Teflon ring on top of each sample to prevent them from floating. The volume of the dissolution medium used was $1 \mathrm{ml}$. At selected intervals of time, doxorubicin concentration was determined by a spectrophotometer (Ultraspec 1000E, Pharmacia Biotech, Sweden) at $233 \mathrm{~nm}$ [15]. All the data were averages of six independent experiments.

\subsection{Modeling of drug release experiments}

To evaluate the performance of doxorubicin loaded in particulate membranes, a two-step release model was proposed for the analysis of the doxorubicin release from particulate membranes. The distribution of drugs in the membrane is divided into two fractions: 
the interparticulate matrix and the intraparticulate matrix, i.e. drugs exists between and within the EVAL particles, each with its respective mass transfer coefficient. Therefore, theoretical analysis describing doxorubicin release from particulate membranes to the medium was considered to be two independent processes. Due to the dense particle structure [4], the doxorubicin transferred from the intraparticulate matrix to the interparticulate matrix is assumed to be far small in comparison with the doxorubicin transferred from the interparticulate matrix to the medium. Thus, the release of doxorubicin from the interparticulate matrix to the medium is the first step and the release of doxorubicin from the intraparticulate matrix to the medium is neglected until the time lag $(T)$. In addition, the drug concentrations in the medium $\left(C_{\mathrm{m}}\right)$ and in the interparticulate matrix $\left(C_{1}\right)$ are assumed to be uniform. At the first step: $0<t<T$, the release of doxorubicin from the interparticulate matrix to the medium is given by

$V_{\mathrm{m}} \frac{\mathrm{d} C_{\mathrm{m}}}{\mathrm{d} t}=k_{1} A_{1}\left(C_{1}-C_{\mathrm{m}}\right)$

where $V_{\mathrm{m}}$ is the volume of the medium, $A_{1}$ the membrane area and $k_{1}$ is the mass transfer coefficient of drug in the interparticulate matrix through interconnected network surrounding particles to the medium. For the sake of simplicity, we treated $k_{1}$ as a lumped mass transfer coefficient containing the effect of drug partition, drug diffusion in the membrane and in the hydrodynamic diffusion layer. The values used were $V_{\mathrm{m}}=1 \mathrm{~cm}^{3}$ and $A_{1}=1.77 \mathrm{~cm}^{2}$. Eq. (1) can be solved analytically to give

$C_{\mathrm{m} t}=C_{10}\left(1-\mathrm{e}^{-\alpha t}\right)\left(\frac{V_{1}}{V_{1}+V_{\mathrm{m}}}\right)$

where $\alpha=k_{1} A_{1}\left[\left(1 / V_{1}\right)+\left(1 / V_{\mathrm{m}}\right)\right] . C_{10}$ and $C_{\mathrm{m} t}$ refers, respectively, to the initial drug concentration in the interparticulate matrix and the drug concentration in the medium at time $t$, respectively. $V_{1}$ is the volume of interparticulate matrix which equals the volume of membrane minus the volume of total particles. The value used was $V_{1}=4.5 \times 10^{-3} \mathrm{~cm}^{3}$. (The volume of total particles is further discussed in the next section.)

The second step $(t>T)$ is diffusion of doxorubicin from the intraparticulate matrix through the interparticulate matrix into the medium. Likewise, the drug concentration in the intraparticulate matrix $\left(C_{2}\right)$ was assumed to be uniform. Thus, the mass balance for

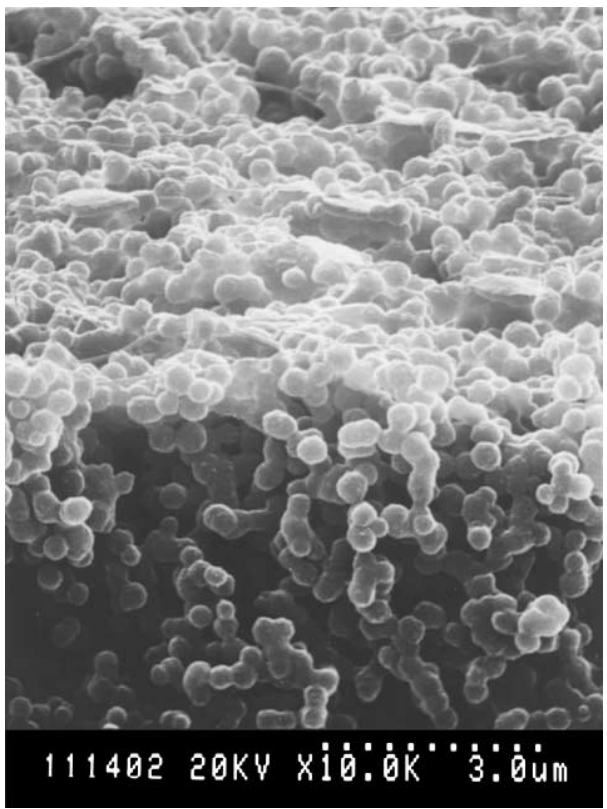

Fig. 1. SEM photograph of a particulate EVAL membrane loaded with doxorubicin.

drug between the intraparticulate and the interparticulate matrix is given as

$V_{2} \frac{\mathrm{d} C_{2}}{\mathrm{~d} t}=-k_{2} A_{2}\left(C_{2}-C_{1}\right)$

where $k_{2}$ is the mass transfer coefficient of drug in the intraparticulate matrix, $A_{2}$ the effective transfer area of total particles and $V_{2}$ is the volume of total particles. The volume of total solid particles is assumed to equal the weight of membrane divided by the density of EVAL. The number of particles equals the volume of total particles divided by the volume of a particle. The diameter of a particle used was $0.4 \mu \mathrm{m}$ (estimated from Fig. 1), so the effective transfer area of total particles equals the surface area of a particle multiplied by the number of particles. The values used were $A_{2}=$ $1636.2 \mathrm{~cm}^{2}$ and $V_{2}=0.011 \mathrm{~cm}^{3}$.

Since drug released form the intraparticulate matrix to the interparticulate matrix will further diffuse into the medium, the mass balance of the whole system gives

$C_{2 t} V_{2}=C_{20} V_{2}-\left(C_{\mathrm{m} t}-C_{\mathrm{m} T}\right) V_{\mathrm{m}}-\left(C_{1 t}-C_{1 T}\right) V_{1}$ 
where $C_{20}$ and $C_{2 t}$ refers, respectively, to the initial drug concentration of the intraparticulate matrix and that at time $t . C_{1 t}, C_{1 T}$ and $C_{\mathrm{m} T}$ refers to the drug concentration in the interparticulate matrix and medium at time $t$ and $T$, respectively.

Substitution of Eqs. (2) and (4) into Eq. (3), yields the drug concentration in the medium at $t>T$, a typical release pattern, with a continuous release over several hours. This system released $261 \pm 25 \mathrm{ng}$ of doxorubicin for the $8 \mathrm{~h}$. However, the particulate membranes show an irregular release profile as a function of time. Approximately $270 \pm 22 \mathrm{ng}$ of doxorubicin was released in the first

$C_{2 t}=\frac{C_{20}\left(1-\mathrm{e}^{-\beta(t-T)}\right)+C_{1 T}\left(\left(V_{1} / V_{2}\right)+\mathrm{e}^{-\beta(t-T)}\right)+C_{\mathrm{m} T}\left(V_{\mathrm{m}} / V_{2}\right)-\left(C_{1 T}-C_{2 T}\right)\left(1+V_{1} / V_{2}\right) \mathrm{e}^{-\alpha(t-T)}}{\left(1+\left(V_{1} / V_{2}\right)+V_{\mathrm{m}} / V_{2}\right)}$

where $\beta=k_{2} A_{2}\left[\left(1 / V_{2}\right)+\left(1 / V_{1}\right)\right]$.

\section{Results and discussion}

\subsection{Morphology of membranes}

In this study, the drug release systems were used in the form of a membrane loaded with doxorubicin. The analyses of dense and particulate membrane morphologies were evaluated using SEM. No voids were found in the dense membrane under the observable detection sensitivity of the SEM (not shown here). In Fig. 1, particulate morphologies are shown, which was uniform characterized by a packed bed of particles approximately $0.4 \mu \mathrm{m}$ in diameter. However, particles did not coalesce completely to form a skin layer on the particulate membrane surface. There are two types of pores in such a membrane: large micron-order pores surrounding the particles interconnected into continuous yet tortuous channels and small nano-order pores inside the EVAL particles [4]. Because both types of pores are available to the migration of drug molecules, the drug release behavior of this membrane should differ from ordinary dense membranes.

\subsection{Drug release study}

The target of this research was to investigate release of drug from particulate membranes for designing a new drug delivery system. For comparison purposes, both of the release curves for the dense and particulate membranes were measured (Fig. 2). All experiments were repeated six times, and results are expressed as mean \pm S.D. The dense membranes loaded with doxorubicin seemed to follow
$2 \mathrm{~h}$. This is consistent with particles in the membrane surface did not coalesce so completely that doxorubicin can be easily released into the medium. The burst-release was followed by a plateau release about $2 \mathrm{~h}$. If the release experiment stopped at this time, doxorubicin was released in a simple but relatively fast manner. Nevertheless, when the release experiment was performed continuously, another significant increase in drug delivery took place, during which about $130 \pm 20 \mathrm{ng}$ of doxorubicin came out of the membrane over the next $4 \mathrm{~h}$. The total release amount of doxorubicin is about $400 \mathrm{ng}$ in the $8 \mathrm{~h}$.

From the results it is clear that the mechanism of doxorubicin release from particulate membranes is in

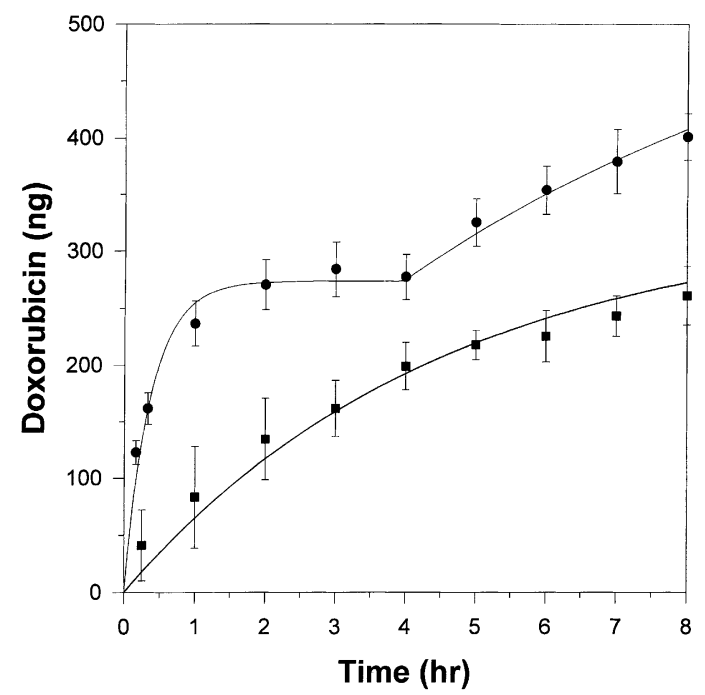

Fig. 2. Release of doxorubicin from EVAL membranes loaded with doxorubicin $(n=6)$. ( $(\mathbf{O})$ Particulate membranes; $(\boldsymbol{\square})$ dense membranes and (-) simulated release from the mathematical model. 
a biphasic pattern, probably due to non-homogeneity of drug distribution in the membrane. The first phase of drug release was a rapid response, which is assumed that some of loaded drugs were accumulated at the voids between EVAL particles during membrane formation. On the other hand, the slower and prolonged second phase is probably due to some of loaded drugs were trapped in EVAL particles during membrane formation. In addition, drug distribution within the particle also can be qualitatively observed by transmission electron microscopy (not shown here).

\subsection{Analysis of drug release data}

Now, we would like to show with the aid of mathematical model mentioned above how the particulate membrane could result into the two-step release curve. As shown in Fig. 2, doxorubicin appeared to be divided into two fractions that were released at time of 0 and $4 \mathrm{~h}$, respectively. Therefore, the drug release data in the first $4 \mathrm{~h}$ were used with Eq. (2) to investigate the characteristics of particulate membranes. The unknown parameters $C_{10}$ and $k_{1}$ were taken as the model parameters. Since the number of experimental data points exceeds the number of parameters to be estimated, we minimized the sum of the squared residuals between the calculated and experimental data to find $C_{10}$ and $k_{1}$. It appeared that an excellent fitting curve matched very closely the experimental data points. The best fitted parameters; $C_{10}$ and $k_{1}$ were adjusted to $61.1 \mu \mathrm{g} / \mathrm{cm}^{3}$ and $1.9 \times 10^{-6} \mathrm{~cm} / \mathrm{s}$, respectively. Therefore, the total amount of doxorubicin loaded in the interparticulate matrix $\left(Q_{1}\right)$, expressed as $V_{1} \times C_{10}$, was about $0.275 \mu \mathrm{g}$. This calculated result closely matched the drug concentration in the medium after the drug release was observed to be stable in the first step. This further assures a reliable calculation.

Subsequently, using Eq. (5), the drug release from the intraparticulate matrix in the second step was simulated. We adopted $T=4 \mathrm{~h}$ as time lag value for drug release in the second step. In the simulation, the best fitted parameters; $C_{20}$ and $k_{2}$ were adjusted to $30.0 \mu \mathrm{g} / \mathrm{cm}^{3}$ and $7.2 \times 10^{-11} \mathrm{~cm} / \mathrm{s}$, respectively. As shown in Fig. 2, release profile in the second step could be well simulated. The simulated drug concentration was in good agreement with the experimental data.

The best fitted $k_{2}$ could be used to investigate drug release kinetics from particulate membranes. It can be seen that the mass transfer coefficient can change by up to 5 orders of magnitude of from the interparticulate matrix to the intraparticulate matrix. However, the drug release in the second step had significant contribution in the total drug release because the total area of particles is relative large. In addition, in order to make comparisons, mass transfer coefficient of doxorubicin from the dense membrane was also estimated from Eq. (2). In the simulation, the volume of the dense membrane used was $2.7 \times 10^{-3} \mathrm{~cm}^{3}$. Fig. 2 shows good agreement between the observed and the simulated release profiles. The initial doxorubicin concentration in the dense membrane and the mass transfer coefficient were taken to be $124.5 \mu \mathrm{g} / \mathrm{cm}^{3}$ and $9.1 \times 10^{-8} \mathrm{~cm} / \mathrm{s}$, respectively. If the dense degree of dense membranes is similar to that of particles of particulate membranes, drug should be released with the similar order of mass transfer coefficient. However, it was observed that drug released from the dense membrane faster than from the particles of particulate membranes. Therefore, although the difference of dense degree in dense membrane and particles of particulate membranes is beyond the observable sensitivity of the SEM, the interstitial cavities in the dense membranes, acting as the access for the transport of drugs through this membrane, are thought to be larger than those within the particles of particulate membranes. This suggests the exact nature of particles has not yet been satisfactorily resolved, but the existence of these dynamic 'molecule pores' can be adequately described in terms of free volume.

Another interesting characteristic of particulate membranes is that the best fitted $C_{20}$ could be used to investigate drug distribution in the particulate membranes. The difference of drug concentration in the intra- and interparticulate matrix defines the driving force for drug diffusion in the second step so the doxorubicin load is one of the most important parameters affecting the release rate. According to the present analysis, although the drug concentration in the intraparticulate matrix was larger than that in the interparticulate matrix after $17 \mathrm{~min}$ of drug release (29.7 $\mu \mathrm{g} / \mathrm{cm}^{3}$, calculated from Eq. (2)), however, the time lag was prolonged to $4 \mathrm{~h}$. A speculative explanation of the release mechanism may be related to the mobility of the medium in the particle. Before allowing drug release from the intraparticulate matrix, the medium must penetrate the EVAL particles to swell the cavities occupied by doxorubicin. This suggests 


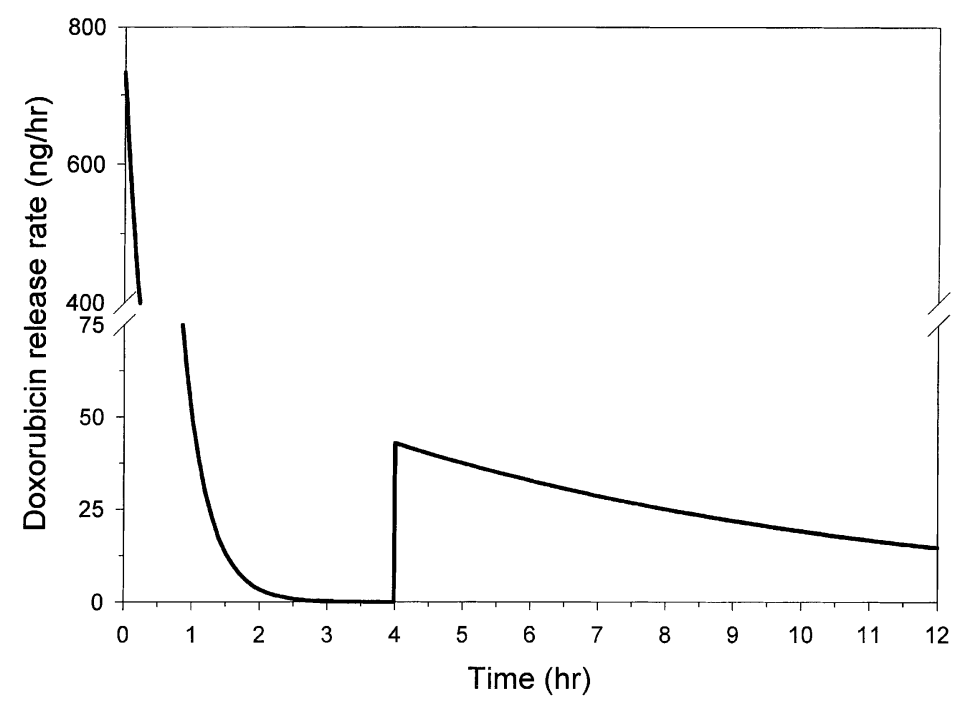

Fig. 3. Release rate of doxorubicin from particulate EVAL membranes.

a completely swelling situation is attained where the drug started in the intraparticulate matrix to diffuse. Thus, swelling, as a result of interaction between the medium and the polymer, is a very important factor in the time lag for drug transport through dense particles.

Furthermore, the total amount of doxorubicin loaded in the intraparticulate matrix $\left(Q_{2}\right)$, expressed as $V_{2} \times C_{20}$, was about $0.330 \mu \mathrm{g}$. Therefore, approximately $55 \%$ of doxorubicin was loaded in the intraparticulate matrix. On basis of the above observation, the incorporation of drug by including into the particles of EVAL membrane has two advantages. Firstly, it shows a better distribution of drug in the intraparticulate matrix. Secondly, the mass transfer coefficient varies by some orders of magnitude with different matrixes in the particulate membranes. Therefore, the outcome supports the concept of utilizing particulate membranes to achieve a shorter response time and a longer drug release system, which means an improvement in the control of doxorubicin delivery. As shown in Fig. 3, the doxorubicin release rate from particulate membranes was plotted as a function of time. This demonstrates quite clearly, that the drug release from particulate membranes is comparable to insulin secreted by pancreas [17]. The first phase of drug release was a rapid but transient response. With continuous exposure to medium, this first response phase faded, later to be followed by a lower and prolonged second phase. In fact, total release of doxorubicin was reached after $96 \mathrm{~h}$ (not shown here).

Furthermore, the present design of particulate membranes, which used 1-octanol to precipitate polymer solution loading drugs, may, limit its use with regard to highly hydrophobic drugs. Hence, the concept of particulate membranes should be considered in the design of any hydrophilic drug delivery system. For example, 1-octanol/water partition coefficient of doxorubicin is 0.52 [18], so doxorubicin may have opportunity partitioning out of the EVAL membranes to be soluble in 1-octanol during membrane formation and hence the incomplete loading doxorubicin compared to the dense membrane. This suggests that EVAL particulate membranes containing drugs with a low water/1-octanol partition coefficient will lose the loaded cargo more quickly. Thus, the ideal loaded drugs in particulate membranes are highly hydrophilic compounds.

In conclusion, as far as polymer membranes are concerned, no investigation has ever been carried out to identify the performance of the particulate structure for a drug delivery system. In the present study, the EVAL membrane with a particulate structure was investigated to control drug release and a two-step release model was applied for the analysis of the biphasic pattern in the drug release experiment. Thus, the EVAL particulate membrane has been used for the first time to investigate drug distribution and release 
characteristics from particulate membranes. Further research about this point may be necessary to make the drug release more versatile and valid in sustained drug release.

\section{Acknowledgements}

Acknowledgment is made to the donors of the Research Fund of Chinese Petroleum Company, administered by the National Science Council of the Republic of China.

\section{References}

[1] S. Loeb, S. Sourirajan, Sea water demineralization by means of an osmotic membrane, Adv. Chem. Ser. 38 (1963) 117.

[2] R.W. Baker, Controlled Release of Biologically Active Agents, Wiley, New York, 1987.

[3] Y. Sakurada, A. Sueoka, M. Kawahashi, Blood purification device using membranes derived from poly(vinyl alcohol) and copolymer of ethylene and vinyl alcohol, Polym. J. 19 (1987) 501.

[4] L.P. Cheng, H.Y. Lin, L.W. Chen, T.H. Young, Solute rejection of dextran by EVAL membranes with asymmetric and particulate morphologies, Polymer 39 (1998) 2135.

[5] D.T. Lin, L.P. Cheng, Y.J. Kang, L.W. Chen, T.H. Young, Effects of precipitation condition on the membrane morphology and permeation characteristics, J. Membr. Sci. 140 (1998) 185.

[6] T.H. Young, C.H. Yao, J.S. Sun, C.P. Lai, L.W. Chen, The effect of morphology variety of EVAL membranes on the behavior of myoblasts in vitro, Biomaterials 19 (1998) 717724.
[7] J.H. Aubert, Isotactic polystyrene phase diagrams and physical gelation, Macromolecules 21 (1988) 3468.

[8] A.M.W. Bulte, B. Folkers, M.H.V. Mulder, C.A. Smolders, Membranes of semicrystalline aliphatic polyamide nylon 4,6: formation by diffusion-induced phase separation, J. Appl. Polym. Sci. 50 (1993) 13.

[9] P. van de Witte, H. Esselbrugge, P.J. Dijkstra, J.W.A. van de Berg, J. Feijen, A morphological study of membranes obtained from the systems polylactide-dioxane-methanol, polylactide-dioxane-water, and polylactide- $\mathrm{N}$-methyl pyrrolidone-water, J. Polym. Sci. Polym. Phys. 34 (1996) 2569.

[10] T.H. Young, J.Y. Lai, W.M. Yu, L.P. Cheng, Equilibrium phase behavior of the membrane forming water-DMSO-EVAL copolymer system, J. Membr. Sci. 128 (1997) 55.

[11] L.P. Cheng, T.H. Young, W.M. You, Formation of crystalline EVAL membranes by controlled mass transfer process in water-DMSO-EVAL copolymer systems, J. Membr. Sci. 145 (1998) 77

[12] T.H. Young, L.P. Cheng, C.C. Hsieh, L.W. Chen, Phase behavior of EVAL polymer in water-2-propanol cosolvent, Macromolecules 31 (1998) 1229.

[13] T.H. Young, C.C. Hsieh, L.Y. Chen, Y.S. Huang, The formation mechanism of membranes prepared from the crystalline EVAL polymer-water (nonsolvent)-2-propanol (nonsolvent) system, J. Membr. Sci. 159 (1999) 21.

[14] W.L. Webber, F. Lago, C. Thanos, E. Mathiowitz, Characteriaation of soluble, salt-loaded, degradable PLGA films and their release of tetracycline, J. Biomed. Mater. Res. 41 (1998) 18.

[15] D.D. Lasic, Doxorubicin in sterically stabilized liposomes, Nature 380 (1996) 561.

[16] R.E. Kesting, Synthetic Polymeric Membranes, Wiley, New York, 1985.

[17] R.M. Berne, M.N. Levy, Principle of Physiology, Wolfe Publishing Limited, 1990.

[18] S.C. Dollery, Therapeutic Drugs, Vol. 1, Churchill Livivgstone, UK, 1995. 